\title{
Cardiometabolic dysregulation and cognitive decline: potential role of depressive symptoms
}

Norbert Schmitz, Sonya S. Deschênes, Rachel J. Burns, Sofia M. Danna, Oscar H. Franco, M. Arfan Ikram, Mika Kivimäki, Archana Singh-Manoux and Henning Tiemeier

\section{Background}

Previous studies have examined associations of cardiometabolic factors with depression and cognition separately.

\section{Aims}

To determine if depressive symptoms mediate the association between cardiometabolic factors and cognitive decline in two community studies.

\section{Method}

Data for the analyses were drawn from the Rotterdam Study, the Netherlands $(n=2940)$ and the Whitehall II study, UK $(n=4469)$.

\section{Results}

Mediation analyses suggested a direct association between cardiometabolic factors and cognitive decline and an indirect association through depression: poorer cardiometabolic status at time 1 was associated with a higher level of depressive symptoms at time 2 (standardised regression coefficient 0.07 and 0.06 , respectively), which, in turn, was associated with greater cognitive decline between time 2 and time 3 (standardised regression coefficient of -0.15 and -0.41 , respectively).

\section{Conclusions}

Evidence from two independent cohort studies suggest an association between cardiometabolic dysregulation and cognitive decline and that depressive symptoms tend to precede this decline.

\section{Declaration of interest}

None.

\section{Copyright and usage}

(c) The Royal College of Psychiatrists 2018.
Cognitive decline is an important public health issue. ${ }^{1}$ Progressive deterioration of cognitive function can affect an individual's overall health and well-being, including daily self-care activities (for example dressing, bathing and housework), as well as the ability to effectively manage existing medical conditions and to participate actively in society. Increasing evidence suggests that cardiometabolic factors, including central obesity, ${ }^{2}$ dyslipidemia, ${ }^{3}$ hypertension, ${ }^{4}$ insulin resistance or diabetes ${ }^{5}$ and inflammation ${ }^{6}$ might accelerate cognitive decline in midlife and at older ages. Similarly, the metabolic syndrome, a cluster of metabolic abnormalities that increase cardiovascular risk, has been associated with cognitive decline in prospective cohort studies. $^{7}$

Emerging evidence suggests that the association between cardiometabolic dysregulation and cognitive decline may include indirect pathways via depressive symptoms. ${ }^{8}$ Specifically, a metaanalysis by Pan et $a l^{9}$ found evidence for a bidirectional association between metabolic syndrome and depression. There is also considerable evidence linking depression with cognitive impairment. Meta-analyses suggest that depression at older ages may be a risk factor for Alzheimer's disease ${ }^{10}$ and have found moderate impairment in executive function, memory and attention in individuals with depression relative to controls. ${ }^{11}$ However, recurrent or increasing levels of depressive symptoms may also be a prodrome of dementia. ${ }^{12}$ We hypothesise that depressive symptoms are one pathway that links cardiometabolic factors to cognitive decline. Given that previous studies have examined associations of cardiometabolic factors with depression and cognitive functioning separately, the temporal relationships between these factors are unknown. Using data from two longitudinal community-based samples of adults in the Netherlands (Rotterdam Study) and the UK (Whitehall II study), this study evaluated if depressive symptoms mediate the association between cardiometabolic factors and cognitive decline while controlling for other risk factors.

\section{Method}

\section{Design/setting and participants}

\section{Rotterdam study}

The Rotterdam Study is a prospective population-based cohort study of adults in Rotterdam, the Netherlands. More details are provided elsewhere. ${ }^{13}$ Briefly, in 1990 all inhabitants aged 55 years and older living in a catchment area in Rotterdam were invited to participate. A total of 7983 (78\%) individuals agreed to participate. The initial cohort was expanded by 3011 individuals aged 55 years and older in 2000. This study used the third data wave of the first cohort (1997-99) and the first wave of the expanded cohort (2000-01) as baseline. All participants underwent home interviews and an extensive set of examinations in a research centre at baseline and after approximately 5 and 12 years (2002-04 and 2009-11; original cohort) and after approximately 4 and 11 years (2004-05 and 2011-12; extension cohort). The Rotterdam Study has been approved by a medical ethics committee, in accordance with the Population Screening Act: Rotterdam Study, executed by the Netherlands Ministry of Health, Welfare and Sports. All participants in the present analysis provided written informed consent.

Depression was assessed using a validated Dutch 20-item version of the Center for Epidemiologic Studies Depression (CES-D) scale, ${ }^{14}$ which measures self-reported frequency of depressive symptoms experienced during the past week. The CES-D is scored on a four-point Likert scale, ranging from 'rarely or none of the time' to 'most or all of the time'. Cognitive function was assessed twice (second and third wave) at separate centre visits. ${ }^{15}$ The test battery included the Stroop test, ${ }^{16}$ letter-digit substitution task (LDST), ${ }^{15}$ verbal fluency test (VF), ${ }^{17} 15$-word verbal learning test $(15-\mathrm{WLT})^{18}$ and the Purdue pegboard test. ${ }^{19}$ Higher scores on each test indicate a better performance, except for the Stroop test in which a higher score indicates a worse performance. Scores for the Stroop test were therefore inverted. All 
tests were scored so that higher scores indicated better cognitive function.

Cardiometabolic dysregulation was defined using the facets of the metabolic syndrome: ${ }^{20}$ elevated blood pressure $(\mathrm{BP}>130 / 85 \mathrm{mmHg}$ or use of antihypertensive medication), impaired glycaemic control (fasting blood glucose concentration greater than $5.6 \mathrm{mmol} / \mathrm{L}$ (38 $\mathrm{mmol} / \mathrm{mol}$ ) or diagnosed type 2 diabetes), low high-density lipoprotein cholesterol $(<1.03 \mathrm{mmol} / \mathrm{L}$ in men and $<1.30 \mathrm{mmol} / \mathrm{L}$ in women), elevated triglycerides $(>1.7 \mathrm{mmol} / \mathrm{L})$ and central obesity (waist circumference $\geq 102 \mathrm{~cm}$ (men), $\geq 88 \mathrm{~cm}$ (women)). Systemic inflammation was added as an additional risk factor (C-reactive protein $>3 \mathrm{mg} / \mathrm{L}$ ). These measures were collected during research centre visits.

Level of education was categorised into low (primary education), medium (intermediate general education) and high (higher vocational education or university) levels. Smoking status was assessed by interview and categorised as smoker (current smoker) and non-smoker (never or former smoker). Physical activity levels were assessed with an adapted version of the Zutphen Physical Activity Questionnaire, which assigns a metabolic equivalent of task (MET) to all activities. ${ }^{21}$ Participants were classified into three groups of physical activity as low physical activity $(<10$ Methours per week); medium physical activity (between 10 and 50 Met-hours per week) and high physical activity ( $>50$ Met-hours per week).

\section{The Whitehall II study}

The Whitehall II study is a prospective cohort study of Londonbased office staff working in 20 civil service departments. More details are provided elsewhere. ${ }^{22}$ Briefly, a total of 10308 individuals (73\% response rate) aged 35-55 years were recruited between 1985 and 1988. Ten follow-up assessments were conducted; participants were assessed approximately every 2 to 3 years. A clinical assessment was conducted every second phase. Informed consent was obtained from all participants, and the University College London Medical School Committee on the Ethics of Human Research approved the protocol.

Wave five (1997-1999) was used as the baseline for our analyses where the age range was between 45 and 69 years. Cardiometabolic factors were categorised as in the Rotterdam Study. Missing waist circumference was substituted with body mass index (BMI $>30 \mathrm{~kg} / \mathrm{m}^{2}$ for obesity). Depression was assessed using the 20-item CES-D scale, ${ }^{14}$ which was administered at wave seven for the first time. Therefore, we have used the four-item depression subscale of the 30 -item General Health Questionnaire $(\mathrm{GHQ})^{23}$ to control for depressive symptoms at baseline.

Educational attainment was grouped into three levels (no formal education or lower secondary education, intermediate education and higher degree). Ethnicity was drawn from wave one and categorised as White/Black minority ethnic. Smoking status was assessed by interview and coded as never, former and current. Intensity of physical activity was assessed via questionnaires. Participants were asked about the frequency and duration of their participation in 'mildly energetic', 'moderately energetic' and 'vigorous' physical activity. The measures were combined and categorised high, medium and low activity.

Cognitive function was assessed by four standard tasks at waves five, seven and nine. Short-term verbal memory was assessed with a 20 -word free recall test. The Alice Heim 4-I (AH4-I) ${ }^{24}$ was used to assess inductive reasoning. Phonemic and semantic verbal fluency was assessed via 'S' words for phonemic fluency and via 'animal' words for the semantic fluency. ${ }^{25}$ More details can be found elsewhere. ${ }^{26}$ The most recent waves (i.e. seven and nine) were used in the present study.

\section{Statistical analyses}

Structural equation modelling was used to examine a potential mediating role of depression in the association between cardiometabolic dysregulation and cognitive decline. Mediation analyses requires a temporal relationship between exposure (cardiometabolic dysregulation, wave one), mediator (depressive symptoms, wave two) and outcome (cognitive decline, wave three). Therefore, longitudinal data from three waves was used for each cohort. ${ }^{27}$ Structural equation models are an extension of regression analysis that can handle measurement error, latent variables and relationships among latent and observed variables. Latent variables summarise the information from observed variables and account for measurement error and the individual contribution of each measure. ${ }^{28}$ Elevated blood pressure, impaired glycaemic control, low highdensity lipoprotein cholesterol, elevated triglycerides, central obesity and systemic inflammation were used as indicators for the baseline latent cardiometabolic dysregulation measure. The cognitive tests in each study were used as indicators for a general cognitive function (g-factor).

Change in cognitive function between two assessments was modelled using a latent change score approach. ${ }^{29}$ In these models, a latent difference score is created by a distinct latent construct representing the difference between the two cognitive g-factors. Briefly, the model constrains the association between the first latent score and the second latent score to 1 such that any variance in the second latent score that was not explained by the first latent score would be accounted for by the latent change score for a given participant.

The conceptual framework is shown in Fig. 1: first, two latent constructs representing the cognitive g-factors at time $2\left(T_{2}\right)$ and time $3\left(T_{2}\right)$ were created. Second, a latent change score (cognitive decline) was constructed so that the cognitive g-factor at $T_{3}$ is considered to be the sum of the cognitive g-factor at $T_{2}$ plus the latent change score. Regression coefficients between the $T_{2}$ and $T_{3}$ cognitive functioning $\mathrm{g}$-factors were constrained to 1 , and regression coefficients between the $T_{3}$ cognitive functioning g-factor and the latent change score was also constrained to 1 so that the latent change score represented the nature of the change in cognitive functioning g-factors from $T_{2}$ to $T_{3}$. The cognitive g-factor at $T_{2}$ was standardised to a mean of 1 and a s.d. of 1 so that higher scores indicate better cognitive functioning. Third, the latent change score was correlated with the cognitive functioning g-factors from $T_{2}$ to account for individual changes over time that are associated with the initial cognitive functioning score at $T_{2}$. A negative change score indicates a cognitive decline.

Depressive symptoms at baseline were included in all models to account for previous depression symptoms. In a final step, associations between cardiometabolic risk factor at time $1\left(T_{1}\right)$ (latent variable), depression at $T_{2}$ (manifest variable) and cognitive change (latent variable) are modelled using a regression framework. We controlled for gender, age, education, ethnicity, smoking and physical activity in our analyses. Depressive symptoms at baseline were included in all models to account for previous depression symptoms. In the Rotterdam Study, the cohort source was also controlled for.

Depression was modelled as a mediator between cardiometabolic dysregulation and cognitive decline. A direct association between the metabolic risk factor at $T_{1}$ and cognitive decline $\left(T_{2}-T_{3}\right)$ was estimated, as well as an indirect association through depression at $T_{2}$ (for example metabolic risk factor at $T_{1}$ predicts depression at $T_{2}$, which in turn predicts cognitive decline from $T_{2}$ to $T_{3}$ ). Indirect association coefficients were calculated by multiplying the standardised coefficients for the paths from the predictor to the mediator (for example metabolic risk factor to depression) and from the mediator (depression) to the outcome (cognitive decline). ${ }^{30}$ 


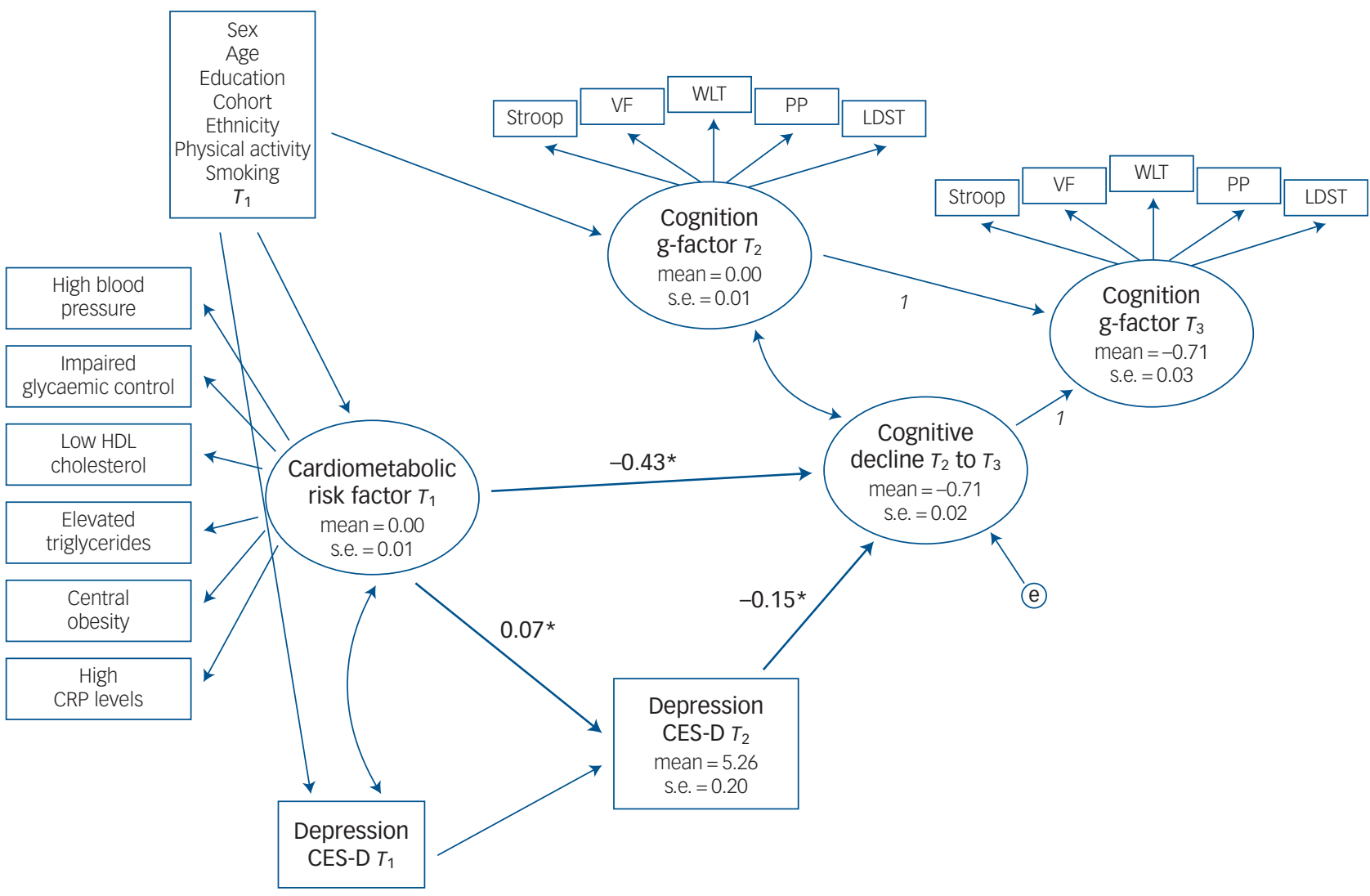

Fig. 1 Depressive symptoms as a potential mediator between cardiometabolic risk factors at baseline and cognitive decline at follow-ups in the Rotterdam Study cohort.

Rectangles represent measured variables, and circles represent latent constructs. Standardised regression coefficients are presented. Italicised path coefficients reflect constraints imposed on the model. The second assessment (time $2, T_{2}$ ) was conducted approximately $4-5$ years after baseline and the third assessment (time $3, T_{3}$ ) was conducted approximately 10-11 years after baseline. The variable 'cardiometabolic risk factors' is standardised so that a positive score indicates higher risk. The variable 'cognitive decline' is standardised so that positive scores indicate cognitive improvement from $T_{2}$ to $T_{3}$ and negative scores indicate cognitive decline from $T_{2}$ to $T_{3} . T_{1}$, time 1; VF, verbal fluency test; WLT, word verbal learning test; PP, Purdue pegboard test; LDST, letter-digit substitution task; HDL, high-density lipoprotein; CRP, C-reactive protein; CES-D, Center for Epidemiologic Studies Depression scale. ${ }^{*} P<0.05$.

The general goodness of fit of each model was evaluated using the comparative fit index (CFI) and the root mean square error of approximation (RMSEA). A CFI of 0.90 or more and RMSEA of 0.08 or lower indicates adequate fit. ${ }^{31}$ Multiple imputed values for the missing data from the variables used in the analysis were generated (PROC MI in SAS). In sensitivity analyses, analyses were rerun without imputed values for missing covariates. All analyses were conducted with SAS (version 9.4) and MPlus (version 7.4). Because observed cardiometabolic indicators were dichotomous, the weighted least squares estimator with a diagonal weight matrix, robust standard errors and a mean- and variance-adjusted $\chi^{2}$ test statistic (WLSMV) and theta parameterisation ${ }^{32}$ were used for parameter estimation in MPlus.

\section{Results}

\section{Findings from the Rotterdam study}

A total of 2940 individuals participated in all three assessments of the Rotterdam Study and had data on cardiometabolic factors, depression and cognitive functioning (see flow chart for sample selection in supplementary Fig. 1; available at http://dx.doi.org/ 10.1192/bjp.2017.26). Mean ages at baseline, first and second follow-ups were 65.0 years $($ s.d. $=5.9), 69.3$ years $($ s.d. $=6.1)$ and 75.8 years $($ s.d. $=6.1)$, respectively. There were more women than men in the sample (57\%).
Table 1 presents baseline characteristics of participants. There were sociodemographic and health differences at baseline between those who participated in all three assessments and those who participated only in one or two assessments; individuals who participated at all assessments were younger (65 v. 73 years), more likely to have a high educational level (16.0 v. 10.6\%), more likely to have a high physical activity level ( $74.6 v 59.1 \%)$, less likely to have elevated depressive symptoms $(5.8 v .15 .3 \%)$ and were more likely to have no metabolic risk factors (15.4 v. 9.6\%). See supplementary Fig. 1 for a flow chart of the sample selection.

Mean depression scores increased between the first (mean 3.9, s.d. $=6.2$ ) and second assessment (mean 5.3, s.d. $=6.9 ; P<0.001$ ). Individuals with no metabolic risk factor had lower mean depression scores at the second assessment (mean 4.5, s.d. $=5.7$ ) than those with one or two metabolic risk factors (mean 5.2, s.d. =6.8) and those with three or more metabolic risk factors (mean 5.7, s.d. $=7.5$ ) (test for a linear trend across the cardiometabolic risk factors groups: $F(1,2937)=13.4, P<0.001)$

Cognitive decline was observed between the second and third assessment on all cognitive functioning subtests: the effect sizes of change (difference between first and second cognitive functioning subtest score, divided by standard deviation of the first score) were $0.41,0.18,0.39,0.12$ and 0.50 for the letter-digit substitution task, verbal fluency test, Stroop test, 15-word verbal learning test and Purdue pegboard test, respectively.

Table 2 describes the associations between the number of cardiometabolic risk factors at baseline and change in cognitive 
Table 1 Baseline characteristics of the Rotterdam Study cohort and the Whitehall II Study cohort

\begin{tabular}{|c|c|c|}
\hline & $\begin{array}{c}\text { Rotterdam } \\
\text { Study }(n=2940)\end{array}$ & $\begin{array}{c}\text { Whitehall II } \\
\text { Study }(n=4635)\end{array}$ \\
\hline Age, mean (s.d.) & $65.0(5.9)$ & $55.2(5.9)$ \\
\hline \multicolumn{3}{|l|}{ Gender, \% } \\
\hline Women & 57.0 & 26.1 \\
\hline \multicolumn{3}{|l|}{ Educational level, \% } \\
\hline Low & 7.5 & 31.9 \\
\hline Intermediate & 76.4 & 31.1 \\
\hline High & 16.0 & 37.0 \\
\hline \multicolumn{3}{|l|}{ Ethnicity, \% } \\
\hline White & 98.4 & 93.3 \\
\hline \multicolumn{3}{|l|}{ Smoking, \% } \\
\hline Current smoker & 19.0 & 8.0 \\
\hline \multicolumn{3}{|l|}{ Physical activity, \% } \\
\hline Low & 0.7 & 14.1 \\
\hline Medium & 24.7 & 32.1 \\
\hline High & 74.6 & 53.9 \\
\hline \multicolumn{3}{|l|}{ Cardiometabolic risk factors } \\
\hline Hypertension, \% & 51.0 & 38.0 \\
\hline Impaired glycaemic control, \% & 43.5 & 15.9 \\
\hline $\begin{array}{l}\text { Low high-density lipoprotein } \\
\text { cholesterol, \% }\end{array}$ & 27.2 & 14.8 \\
\hline Elevated triglycerides, \% & 30.6 & 24.2 \\
\hline Elevated CRP levels, \% & 26.5 & 16.2 \\
\hline Central obesity, \% & 41.7 & 17.6 \\
\hline \multicolumn{3}{|l|}{ Depression } \\
\hline CES-D summary score & $3.9(6.2)$ & \\
\hline Elevated depressive symptoms, \% & 5.8 & \\
\hline $\begin{array}{l}\text { GHQ depression subscale summary } \\
\text { score }\end{array}$ & & $0.9(1.8)$ \\
\hline Elevated depressive symptoms \% & & 16.5 \\
\hline \multicolumn{3}{|c|}{$\begin{array}{l}\text { CRP, C-reactive protein; CES-D, Center for Epidemiologic Studies - Depression; GHQ, } \\
\text { General Health Questionnaire. }\end{array}$} \\
\hline
\end{tabular}

functioning (g-factors controlled for age and gender). Individuals with no cardiometabolic risk factors had the highest cognitive functioning scores at $T_{2}$ and $T_{3}$ whereas individuals with three or more cardiometabolic risk factors had the lowest cognitive functioning scores at $T_{2}$ and $T_{3}$. The largest cognitive decline was observed for those with three or more cardiometabolic risk factors.

Results of the mediation analyses are presented in Fig. 1. The model fit was acceptable $(\mathrm{CFI}=0.933$, RMSEA $=0.041)$. Results show that a poorer cardiometabolic status at baseline was associated with greater cognitive decline, after controlling for age, gender, education, ethnicity, cohort, smoking and physical activity. There was a direct association from the latent variable cardiometabolic risk factor to cognitive decline (standardised regression coefficient of $-0.43, P<0.001)$ and an indirect association through depression; poorer cardiometabolic status at baseline was associated with a higher level of depressive symptoms at $T_{2}$ (standardised regression coefficient of $0.07, P<0.001$ ) which in turn was associated with cognitive decline (standardised regression coefficient of -0.15 , $P<0.001)$.

The indirect association coefficients were small but significant: -0.011 ( $95 \%$ CI -0.002 to -0.020 ), suggesting that depression partially mediated the association between cardiometabolic status and cognitive decline.

\section{Findings from the Whitehall II study}

A total of 4469 individuals participated in waves five, seven and nine of the Whitehall II study and had data on cardiometabolic factors, depression and cognitive functioning. The mean age at baseline (wave five, mean 55.2, s.d. $=5.9$ ) was almost 10 years lower compared with the Rotterdam Study and there were almost three times more men than women in this cohort. Mean ages at wave seven and nine were 61 (s.d. $=6.0)$ years and $66($ s.d. $=6.0)$ years, respectively.

Table 1 shows sociodemographic and clinical characteristics of participants at wave five (the baseline). Those who participated in all three assessments were more often male $(73.9 \%$ v. $63.3 \%)$, more likely to be younger ( $55 v .57$ years), more likely to have a high educational level $(38.6 \%$ v. $30.7 \%)$, more likely to have a high physical activity level (56.5\% v. 47.8\%), less likely to have elevated depressive symptoms (16.1 v. $20.5 \%)$, and were more likely to have no metabolic risk factors $(31.7$ v. $23.5 \%)$ than those who participated only in wave five. Individuals with no metabolic risk factor had lower mean depression scores at the second assessment (mean 7.7, s.d. $=7.5$ ) then those with three or more metabolic risk factors (mean 8.4, s.d. $=7.8$ ). See supplementary Fig. 1 for a flow chart of the sample selection.

Cognitive decline was observed between the second and third assessment on all cognitive functioning subtests: the effect sizes were $0.07,0.29,0.16$, and 0.14 for the Alice Heim 4-I task, shortterm verbal memory task, phonemic and semantic verbal fluency tasks, respectively. Associations between the number of cardiometabolic risk factors at baseline and the change in cognitive functioning (g-factors controlled for age and gender) are presented in Table 2. Associations were similar as in the Rotterdam Study, although changes in cognitive functioning g-factors were smaller. Individuals with no cardiometabolic risk factors had the highest cognitive functioning scores at $T_{2}$ and $T_{3}$ compared with those with one or more cardiometabolic risk factors.

The mediation analyses (Fig. 2) also suggested a potential mediating effect of depression in the association between metabolic risk factors and cognitive decline. Model fit was acceptable (CFI $=0.917$, RMSEA $=0.049$ ). There was a direct association from the latent variable cardiometabolic risk factor to cognitive decline (standardised regression coefficient of $-0.38 P<0.001)$ and an indirect association through depression: poorer cardiometabolic status at baseline was associated with a higher level of depressive symptoms at $T_{2}$ (standardised regression coefficient of $0.06, P=0.003$ ) which,

\begin{tabular}{|c|c|c|c|c|c|c|c|c|}
\hline \multirow[b]{2}{*}{$\begin{array}{l}\text { Baseline } \\
\text { cardiometabolic risk } \\
\text { factors, } n\end{array}$} & \multicolumn{4}{|c|}{ Rotterdam study } & \multicolumn{4}{|c|}{ Whitehall II study } \\
\hline & $n$ & $\begin{array}{l}\text { Cognitive } \\
\text { functioning } \\
\text { g-factor } \\
T_{2} \text {, mean (s.d.) }\end{array}$ & $\begin{array}{l}\text { Cognitive } \\
\text { functioning } \\
\text { g-factor } \\
T_{3} \text {, mean (s.d.) }\end{array}$ & $\begin{array}{c}\text { Difference } \\
\text { g-factor, mean } \\
\text { (s.d.) }\end{array}$ & $n$ & $\begin{array}{l}\text { Cognitive } \\
\text { functioning } \\
\text { g-factor } \\
T_{2,} \text { mean (s.d.) }\end{array}$ & $\begin{array}{l}\text { Cognitive } \\
\text { functioning } \\
\text { g-factor } \\
T_{3} \text {, mean (s.d.) }\end{array}$ & $\begin{array}{c}\text { Difference } \\
\text { g-factor, mean } \\
\text { (s.d.) }\end{array}$ \\
\hline 0 & 454 & $0.23(0.83)$ & $-0.30(1.05)$ & $-0.53(0.29)$ & 1579 & $0.18(0.90)$ & $0.01(0.94)$ & $-0.18(0.10)$ \\
\hline $1-2$ & 1320 & $0.06(0.89)$ & $-0.52(1.14)$ & $-0.58(0.32)$ & 2279 & $-0.08(0.99)$ & $-0.27(1.03)$ & $-0.19(0.11)$ \\
\hline $3-6$ & 1166 & $-0.16(0.86)$ & $-0.81(1.09)$ & $-0.65(0.31)$ & 777 & $-0.14(1.02)$ & $-0.34(1.06)$ & $-0.20(0.11)$ \\
\hline
\end{tabular}




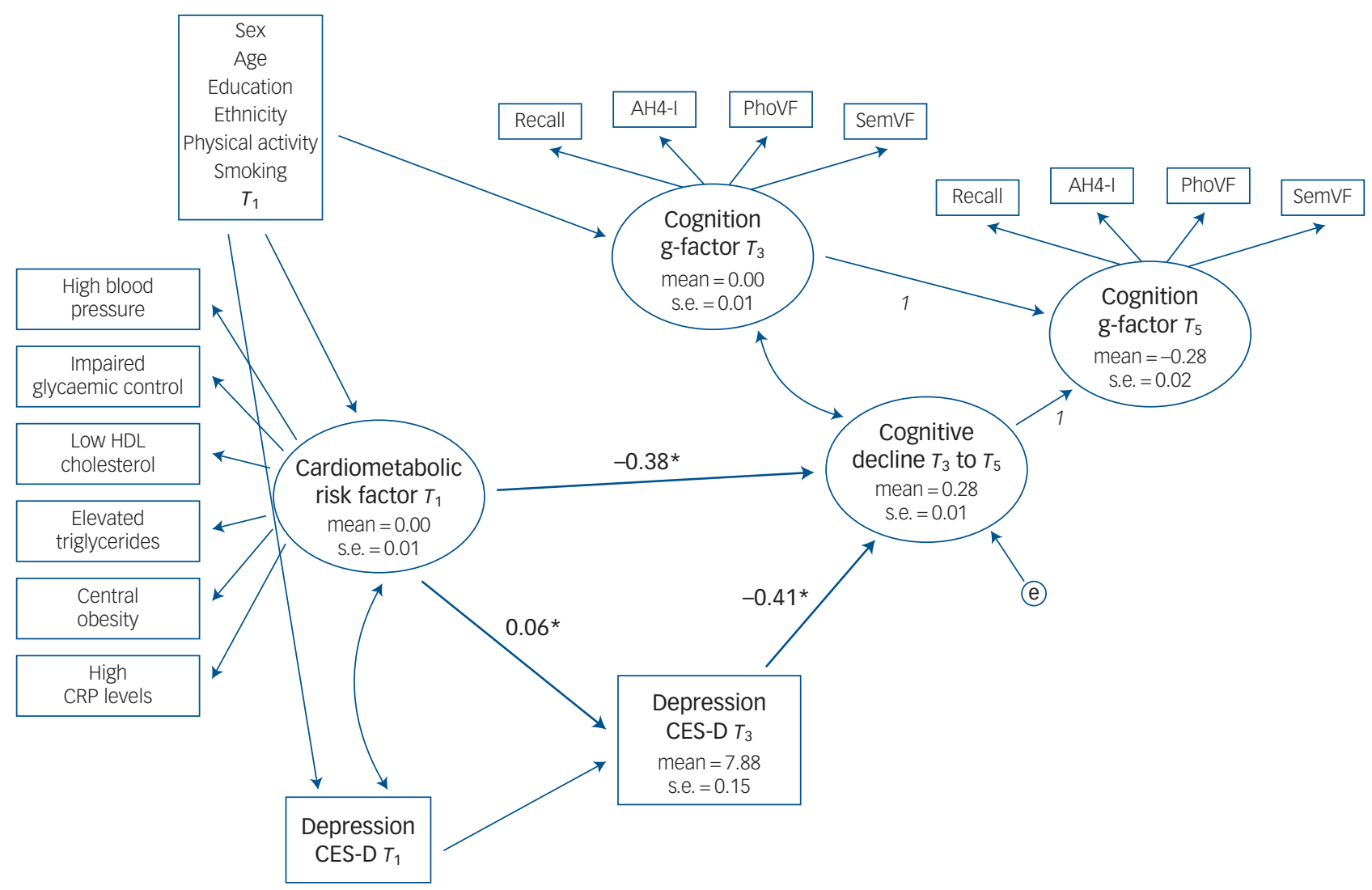

Fig. 2 Depressive symptoms as a potential mediator between cardiometabolic risk factors at baseline and cognitive decline at follow-ups in the Whitehall II study cohort.

Rectangles represent measured variables, and circles represent latent constructs. Standardised regression coefficients are presented (unstandardised estimates shown in parentheses). Italicised path coefficients reflect constraints imposed on the model. The third (time $3, T_{3}$ ) and fifth (time $5, T_{5}$ ) assessment were conducted approximately 4 and 8 years after baseline, respectively. The variable 'cardiometabolic risk factors' is standardised so that a positive score indicates higher risk. The variable 'cognitive decline' is standardised so that positive scores indicate cognitive improvement from to $T_{5}$ and negative scores indicate cognitive decline from $T_{3}$ to $T_{5} . T_{1}$, time 1; AH4-I, Alice Heim 4-I; PhoVF , phonemic verbal fluency; SemVF, semantic verbal fluency; HDL, high-density lipoprotein; CRP, C-reactive protein; CES-D, Center for Epidemiologic Studies Depression scale. ${ }^{\star} P<0.05$.

in turn, was associated with cognitive decline (standardised regression coefficient of $-0.41, P<0.001)$. The indirect association coefficient was -0.023 ( $95 \%$ CI -0.005 to -0.041$)$, which if anything, was higher than in the Rotterdam Study.

\section{Discussion}

\section{Main findings}

This study evaluated temporal associations between cardiometabolic risk factors, depressive symptoms and change in cognitive function in two community studies of individuals aged 45 years and above in the Netherlands and the UK. Evidence from both studies suggest a direct association between cardiometabolic risk factors and cognitive decline and an indirect association through depressive symptoms: cardiometabolic risk factors at $T_{1}$ predicted depressive symptoms at $T_{2}$ (4-5 years later), which, in turn, predicted cognitive decline from $T_{2}$ to $T_{3}$ (4-6 years later).

These results suggest that depression might be one potential pathway through which cardiometabolic dysfunction is associated with cognitive decline. To our knowledge, this is the first study that simultaneously estimated longitudinal associations between cardiometabolic functioning, depressive symptoms and change in cognitive functioning. The congruence of the results from the Rotterdam cohort with those from the Whitehall II cohort, which is comprised individuals 10 -years younger, suggests that the mediating role of depression apply to elderly populations as well as middle-aged adults who are not yet experiencing accelerated cognitive decline.

\section{Strengths and limitations}

The main strength of this study is the repeated measurement of cognitive function using validated cognitive batteries, ${ }^{33}$ as well as the availability of depression assessments and cardiometabolic variables in two large population-based samples. Strengths of the analysis include a structural-equation modelling approach that permitted the modelling of the dynamic relationship between cardiometabolic factors, depressive symptoms and change in cognitive function. This approach accounted for measurement error by including latent variables for cardiometabolic functioning and cognitive functioning. A latent-change score approach for the evaluation of cognitive decline is a more flexible approach than a simple difference-score approach because latent-change score methods separate out the portion of change that is correlated with the initial measurement and thereby removing a source of unreliability of a simple change score.

There are also several study limitations. Depressive symptoms were assessed using a self-report scale, and not diagnostic interviews that assessed depressive symptoms experienced in the past week and does not account for treatment of depression and history of depression. The overall level of depression was low, given that we examined non-clinical cohorts. In addition, lifestyle-related behaviours were also assessed by self-report, and thus may be subject to some 
bias. Cognitive functioning was only assessed at the second and third assessment. Therefore, we were not able to control for baseline cognitive functioning.

Attrition is an expected issue in large elderly population cohorts. Indeed, there were substantial sociodemographic and clinical differences between those who participated in all three assessments and those who did not, indicating that individuals with a better cardiometabolic functioning and a lower level of depressive symptoms were included in our cohort. This might have resulted in an underestimation of the underlying associations.

The time interval ( $4-5$ years) between our single measurement of cardiometabolic functioning and the measurement of cognitive functioning could also be considered as a limitation. For example, Akbaraly et al have demonstrated that only persistent metabolic syndrome was associated with lower cognitive performance in late midlife. ${ }^{26}$ Cardiometabolic functioning might have changed after the assessment because of clinical treatment or change of lifestylerelated behaviours. Finally, the applicability to other ethnic groups may be limited since the majority of the participants in the present study were White.

\section{Comparison with findings from other studies}

Our findings are consistent with previous prospective studies that have focused on comorbid depression as risk factor for cognitive decline or dementia in people with diabetes, a clinical form of cardiometabolic dysregulation. A recent systematic review on the association between depressive symptoms and cognitive functioning in people with diabetes found evidence that the presence of depressive symptoms in people with diabetes was associated with poorer cognitive outcomes. ${ }^{34}$ Sullivan et al ${ }^{35}$ described an interaction between depression and diabetes on cognitive outcomes in the Action to Control Cardiovascular Risk in Diabetes-Memory in Diabetes (ACCORD-MIND) trial; patients with type 2 diabetes and comorbid depression were at higher risk for cognitive decline than those with type 2 diabetes without depression. Similar results have been reported from two studies using administrative databases. ${ }^{36,37}$

Our study adds to this evidence by considering temporality and suggesting that depression might explain some of the association between metabolic factors and cognitive decline (partial mediation). Management of cardiometabolic risk factors requires patient engagement. Depression is associated with poorer self-care behaviours (for example following a healthy diet, not smoking, engaging in exercise and medication adherence), which could worsen the management of the metabolic risk factors course and increase the risk of cognitive decline through a long-term exposure to metabolic risk factors. ${ }^{26}$

Cardiometabolic dysregulations and depression also share common pathophysiological mechanisms, including dysregulation of the hypothalamic-pituitary-adrenocortical (HPA) axis. ${ }^{38}$ The co-occurrence of both conditions might adversely have an impact on both conditions, which can in turn result in several metabolic dysregulations and might amplify the risk of cognitive decline. ${ }^{39}$ Vogelzangs et $a l^{40}$ have shown that metabolic dysregulations predicted a more chronic course of depressive disorders. Recurrent or chronic depressive symptoms are associated with prolonged exposure to psychosocial and other life stresses, which can cause wear and tear to different circulatory, inflammatory, immune and psychological regulatory systems ${ }^{41,42}$ and increase allostatic load. ${ }^{41}$ Allostatic load has been associated with cognitive decline in population studies. ${ }^{43}$ Liu et al ${ }^{44}$ concluded in a recent review that several other overlapping physiological mechanisms in addition to HPAaxis disturbances may explain the association of metabolic dysfunction with worse cognition in individuals with depression, including abnormalities in brain-derived neurotrophic factor signalling, adipose-derived hormones, insulin signalling, inflammatory cytokines, and oxidative and nitrosative stress.

\section{Implications}

In conclusion, our findings show that people with metabolic dysregulation are at increased risk of cognitive decline and that depressive symptoms may partially mediate this decline. This has important implications for investigating the pathways that could link metabolic dysregulation and increased risk of cognitive decline. For adequate prevention of cognitive decline both cardiometabolic and mental health should play a key role.

Norbert Schmitz, PhD, Department of Psychiatry, McGill University, Montreal, Douglas Mental Health University Institute, Montreal and Montreal Diabetes Research Centre, Montreal, Quebec, Canada; Sonya S. Deschênes, PhD, Rachel J. Burns, PhD, Department of Psychiatry, McGill University, Montreal, and Douglas Mental Health University Institute, Montreal Quebec, Canada; Sofia M. Danna, MSc, Department of Epidemiology and Biostatistics, McGill University, Montreal, Quebec, Canada; Oscar H. Franco, MD, PhD, M. Arfan Ikram, MD, PhD, Department of Epidemiology, Erasmus MC-University Medical Center Rotterdam, Rotterdam, the Netherlands; Mika Kivimäki, PhD, Department of Epidemiology and Public Health, University College London (UCL) London, UK: Archana Singh-Manoux PhD Department of Epidemiology and Public Health, University College London (UCL), London, UK and INSERM U1018, Center for Research in Epidemiology and Population Health, Paul Brousse Hospital, Villejuif, France; Henning Tiemeier, MD, PhD, Department of Epidemiology, Department of Psychiatry and Department of Child and Adolescent Psychiatry, Erasmus MC-University Medical Center Rotterdam, Rotterdam, the Netherlands

Correspondence: Norbert Schmitz, Douglas Mental Health University Institute, MCGill University, 6875 LaSalle Boulevard, Montreal, Quebec, H4H 1R3, Canada. Email: norbert. schmitz@mcgill.ca

First received 9 May 2017, final revision 18 Oct 2017, accepted 27 Oct 2017

\section{Funding}

The Rotterdam Study is funded by the Erasmus Medical Center and Erasmus University, Rotterdam, the Netherlands Organization for the Health Research and Development (ZonMw), the Research Institute for Diseases in the Elderly (RIDE), the Ministry of Education, Culture and Science, the Ministry for Health, Welfare and Sports, the European Commission (DG XII), and the Municipality of Rotterdam. Continuing data collection on the Whitehall II study is funded by the Medical Research Council (K013351), National Institute on Aging (AG13196), National Heart Lung and Blood Institute (HL36310) and the British Heart (AG13196), National Heart Lung and Blood Institute (HL36310) and the British Heart
Foundation. No funding was received to complete the analysis and interpretation of data for this paper. The funding agencies had no role in the design or conduct of the study, in the collection, management, analysis, or interpretation of the data, or in the preparation, review, or approval of the manuscript.

\section{Supplementary material}

Supplementary material is available online at https://doi.org/ 10.1192/bjp.2017.26.

\section{References}

1 Jacqmin-Gadda H, Alperovitch A, Montlahuc C, Commenges D, Leffondre K, Dufouil C, et al. 20-Year prevalence projections for dementia and impact of preventive policy about risk factors. Eur J Epidemiol 2013; 28: 493-502.

2 Whitmer RA, Gustafson DR, Barrett-Connor E, Haan MN, Gunderson EP, Yaffe K. Central obesity and increased risk of dementia more than three decades later. Neurology 2008; 71: 1057-64.

3 van Vliet P. Cholesterol and late-life cognitive decline. J Alzheimers Dis 2012; 30: S147-S62.

4 Birns J, Kalra L. Cognitive function and hypertension. J Hum Hypertens 2009; 23: 86-96.

5 Bordier L, Doucet J, Boudet J, Bauduceau B. Update on cognitive decline and dementia in elderly patients with diabetes. Diabetes Metab 2014; 40: 331-7.

6 Tegeler C, O'Sullivan JL, Bucholtz N, Goldeck D, Pawelec G, SteinhagenThiessen $\mathrm{E}$, et al. The inflammatory markers CRP, IL-6, and IL-10 are associated with cognitive function-data from the Berlin Aging Study II. Neurobiol Aging 2016; 38: 112-7. 
7 Dik MG, Jonker C, Comijs HC, Deeg DJH, Kok A, Yaffe K, et al. Contribution of metabolic syndrome components to cognition in older individuals. Diabetes Care 2007; 30: 2655-60.

8 Tuligenga RH, Dugravot A, Tabak AG, Elbaz A, Brunner EJ, Kivimaki M, et al. Midlife type 2 diabetes and poor glycaemic control as risk factors for cognitive decline in early old age: a post-hoc analysis of the Whitehall II cohort study. Lancet Diabetes Endocrinol 2014; 2: 228-35.

9 Pan A, Keum N, Okereke Ol, Sun Q, Kivimaki M, Rubin RR, et al. Bidirectional association between depression and metabolic syndrome a systematic review and meta-analysis of epidemiological studies. Diabetes Care 2012; 35: 1171-80.

10 Ownby RL, Crocco E, Acevedo A, John V, Loewenstein D. Depression and risk for Alzheimer disease - systematic review, meta-analysis, and metaregression analysis. Arch Gen Psychiatry 2006; 63: 530-8.

11 Rock PL, Roiser JP, Riedel WJ, Blackwell AD. Cognitive impairment in depression: a systematic review and meta-analysis. Psychol Med 2014; 44: 2029-40.

12 Mirza SS, Wolters FJ, Swanson SA, Koudstaal PJ, Hofman A, Tiemeier H, et al. 10-year trajectories of depressive symptoms and risk of dementia: a population-based study. Lancet Psychiatry 2016; 3: 628-35.

13 Hofman A, Murad SD, van Duijn CM, Franco OH, Goedegebure A, Ikram MA, et al. The Rotterdam Study: 2014 objectives and design update. Eur J Epidemiol 2013; 28: 889-926.

14 Beekman ATF, Deeg DJH, VanLimbeek J, Braam AW, DeVries MZ, VanTilburg W. Criterion validity of the Center for Epidemiologic Studies Depression scale (CES-D): Results from a community-based sample of older subjects in the Netherlands. Psychol Med 1997; 27: 231-5.

15 Hoogendam YY, Hofman A, van der Geest JN, van der Lugt A, Ikram MA. Patterns of cognitive function in aging: the Rotterdam Study. Eur J Epidemiol 2014; 29: 133-40.

16 Houx PJ, Jolles J, Vreeling FW. Stroop interference: aging effects assessed with the Stroop color-word test. Exp Aging Res 1993; 19: 209-24.

17 Welsh KA, Butters N, Mohs RC, Beekly D, Edland S, Fillenbaum G, et al. The Consortium to Establish a Registry for Alzheimer's Disease (CERAD). Part V. A normative study of the neuropsychological battery. Neurology 1994; 44 609-14.

18 Bleecker ML, Bollawilson K, Agnew J, Meyers DA. Age-related sex-differences in verbal memory. J Clin Psychol 1988; 44: 403-11.

19 Tiffin J, Asher EJ. The Purdue pegboard; norms and studies of reliability and validity. J Appl Psychol 1948; 32: 234-47.

20 Alberti KGMM, Eckel RH, Grundy SM, Zimmet PZ, Cleeman Jl, Donato KA, et al. Harmonizing the metabolic syndrome a joint interim statement of the International Diabetes Federation Task Force on Epidemiology and Prevention; National Heart, Lung, and Blood Institute; American Heart Association; World Heart Federation; International Atherosclerosis Society; and International Association for the Study of Obesity. Circulation 2009; 120: 1640-5.

21 Koolhaas CM, Dhana K, Golubic R, Schoufour JD, Hofman A, van Rooij FJA, et al. Physical activity types and coronary heart disease risk in middle-aged and elderly persons. Am J Epidemiol 2016; 183: 729-38.

22 Marmot M, Brunner E. Cohort profile: the Whitehall II study. Int J Epidemiol 2005; 34: 251-6.

23 Nicholson A, Fuhrer R, Marmot M. Psychological distress as a predictor of CHD events in men: the effect of persistence and components of risk. Psychosom Med 2005; 67: 522-30.

24 Heim AW. AH 4 group test of general intelligence. NFER-Nelson, 1970

25 Borkowski JG, Benton A, Spreen O. Word fluency and brain damage. Neuropsychologia 1967; 5: 135-40.
26 Akbaraly TN, Kivimaki M, Shipley MJ, Tabak AG, Jokela M, Virtanen M, et al. Metabolic syndrome over 10 years and cognitive functioning in late midlife the Whitehall II study. Diabetes Care 2010; 33: 84-9.

27 Kraemer HC, Stice E, Kazdin A, Offord D, Kupfer D. How do risk factors work together? Mediators, moderators, and independent, overlapping, and proxy risk factors. Am J Psychiatry 2001; 158: 848-56.

28. Bollen KA. Structural Equations with Latent Variables. Wiley, 1989.

29 McArdle JJ. Latent variable modeling of differences and changes with longitudinal data. Annu Rev Psychol 2009; 60: 577-605.

30 Preacher KJ, Hayes AF. Asymptotic and resampling strategies for assessing and comparing indirect effects in multiple mediator models. Behav Res Methods 2008; 40: 879-91.

31 Marsh HW. In search of golden rules: Comment on hypothesis-testing approaches to setting cutoff values for fit indexes and dangers in overgeneralizing Hu and Bentler's (1999) findings. Struct Equ Modeling 2004; 11: 320-41.

32 Muthén LKaM B.O. Mplus User's Guide (7th edn). Muthén \& Muthén, 2012.

33 Singh-Manoux A, Fayosse A, Sabia S, Canonico M, Bobak M, Elbaz A, et al. Atrial fibrillation as a risk factor for cognitive decline and dementia. Eur Heart J 2017; 38: $2612-8$.

34 Danna SM, Graham E, Burns RJ, Deschenes SS, Schmitz N. Association between depressive symptoms and cognitive function in persons with diabetes mellitus: a systematic review. PLoS One 2016; 11: e0160809.

35 Sullivan MD, Katon WJ, Lovato LC, Miller ME, Murray AM, Horowitz KR, et al. Association of depression with accelerated cognitive decline among patients with type 2 diabetes in the ACCORD-MIND trial. JAMA Psychiatry 2013; 70 1041-7.

36 Katon WJ, Lin EHB, Williams LH, Ciechanowski P, Heckbert SR, Ludman E, et al. Comorbid depression is associated with an increased risk of dementia diagnosis in patients with diabetes: a prospective cohort study. J Gen Intern Med 2010; 25: 423-9.

37 Katon W, Lyles CR, Parker MM, Karter AJ, Huang ES, Whitmer RA. Association of depression with increased risk of dementia in patients with type 2 diabetes: the Diabetes and Aging Study. Arch Gen Psychiatry 2012; 69: 410-7.

38 Musselman DL, Evans DL, Nemeroff CB. The relationship of depression to cardiovascular disease - Epidemiology, biology, and treatment. Arch Gen Psychiatry 1998; 55: 580-92.

39 Epel ES. Psychological and metabolic stress: a recipe for accelerated cellular aging? Hormones (Athens)- 2009; 8: 7-22.

40 Vogelzangs N, Beekman ATF, Dortland A, Schoevers RA, Giltay EJ, de Jonge P, et al. Inflammatory and metabolic dysregulation and the 2-year course of depressive disorders in antidepressant users. Neuropsychopharmacology 2014; 39: 1624-34.

41 McEwen BS. Mood disorders and allostatic load. Biol Psychiatry 2003; 54: 200-7.

42 Lesage A. Heuristic model of depressive disorders as systemic chronic disease. Epidemiol Psychiatr Sci 2015; 24: 309-11.

43 Juster RP, McEwen BS, Lupien SJ. Allostatic load biomarkers of chronic stress and impact on health and cognition. Neurosci Biobehav Rev 2010; 35: 2-16.

44 Liu CS, Carvalho AF, McIntyre RS. Towards a "metabolic" subtype of major depressive disorder: shared pathophysiological mechanisms may contribute to cognitive dysfunction. CNS Neurol Disord-Drug Targets 2014; 13: 1693-707. 